\title{
Influence of Different Phenolic Copigments on the Color of Malvidin 3-Glucoside
}

\author{
Manuela Gómez-Míguez,${ }^{\dagger}$ Susana González-Manzano,$‡$ \\ M. Teresa Escribano-BAILÓn, ${ }^{\ddagger}$ Francisco J. Heredia, ${ }^{\dagger}$ AND \\ Celestino Santos-Buelga $*$
}

\begin{abstract}
Laboratory of Food Color and Quality, Department of Food Science and Nutrition, Faculty of
Pharmacy, University of Seville, Seville, Spain, and Grupo de Investigación en Polifenoles, Unidad de

Nutrición y Bromatología, Facultad de Farmacia, Universidad de Salamanca, Salamanca, Spain
\end{abstract}

\begin{abstract}
The effectiveness of seven phenolic compounds (catechin, epicatechin, procyanidin B2, caffeic acid, p-coumaric acid, myricitrin, and quercitrin) as copigments of malvidin 3-glucoside, the major anthocyanin in red wines from Vitis vinifera, using a copigment/pigment molar ratio of 1:1 was assayed in model wine solutions under the same conditions $(\mathrm{pH}=3.6,12 \%$ ethanol). The stability of the copigment-pigment complexes formed was studied during a storage period of 60 days at $25^{\circ} \mathrm{C}$. Fristimulus colorimetry was applied for color characterization of the copigmentation process, and HPLC-DAD-MS was used to monitor changes in the composition of the samples. Copigmentation has been found to occur in all cases despite the low copigment/pigment molar ratio used, although the effect was different depending on the compound. Flavan 3-ols appeared as the less effective copigments, procyanidin B2 being even worse than monomeric flavanols, whereas flavonols behaved as the bestones. These latter copigments also induced the most statistically signifieant bathochromic shift in $\lambda_{\text {max. }}$ In the colorimetric analysis, it was observed that the lightness $L^{*}$ of the copigmented solutions increased with time, chroma $C_{2 b}^{*}$ decreased, and the hue $h_{2 a}$ increased. The copigments that produced a greater increase in the hue angle were the monomeric flavan-3-ols, which therefore showed to be the least protective cofactors against browning of the anthocyanin solution during the storage. With the time of storage, the formation of new pigments was observed in the solutions containing flavanols (xanthylium structures) and hydroxycinnamic acids (pyranoanthocyanins), which explains some of the color modifications produced in these solutions. Another relevant observation was that the stability of the anthocyanin was not much improved by most of the assayed copigments, since quite similar degradation rates were observed in the presence and absence of those cofactors.
\end{abstract}

KEYWORDS: Copigmentation; red wines; color; anthocyanins; tristimulus colorimetry

\section{INTRODUCTION}

Copigmentation is a phenomenon which plays a major role in the expression of a wide range of colors provided by anthocyanins in plants, fruits, berries, and food products. It causes stabilization of the colored structural forms of the anthocyanins and consequently enhances their color $(1-3)$. Copigmentation can occur through intramolecular interactions, established between the central anthocyanin chromophore and aromatic acyl residues covalently linked to their glycosyl moieties (intramolecular copigmentation) $(4-6)$, or through intermolecular interactions of the anthocyanins with colorless

* To whom correspondence should be addressed. Celestino SantosBuelga, Unidad de Nutrición y Bromatología, Facultad de Farmacia, Universidad de Salamanca, Campus Miguel de Unamuno, E-37007 Salamanca, Spain. Phone, +34 923 294537; fax, +34 923 294515; e-mail, csb@usal.es.

University of Seville.

Universidad de Salamanca. compounds, either phenolic or not, through weak hydrophobic forces (7). With regard to the original anthocyanin solutions, the spectra of copigmented solutions usually display a shift of the visible $\lambda_{\max }$ toward greater wavelengths (bathochromic effect) coupled with an increase of absorptivity (hyperchromic effect). The amplitude of these effects depends on many factors such as $\mathrm{pH}$, temperature, the nature of both the anthocyanic chromophore and the copigment, the copigment/pigment molar ratio, and the pigment absolute concentration $(8-11)$.

It is assumed that copigmentation should play an important role in the color of young red wines and their evolution during maturation and aging (12). In an attempt to understand the influence of different factors in wine color and its modifications during storage, many studies have been carried out in model wine solutions by different authors. Malvidin 3-glusoside has been the most widely used pigment, because it is the major anthocyanin in red wines, whereas different flavan-3-ols, 


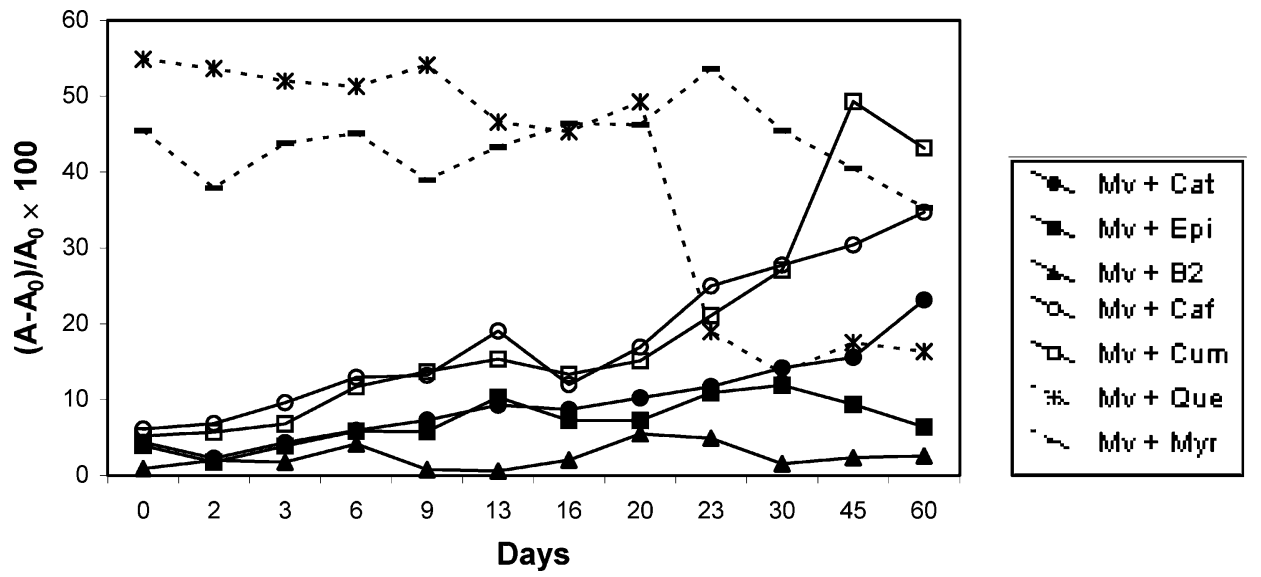

Figure 1. Changes in the ratio $\left[\left(A-A_{0}\right) / A_{0}\right] \times 100$ in the model solutions containing malvidin 3-glucoside and different copigments during storage at 25 ${ }^{\circ} \mathrm{C}$. Abbreviations: Cat, (+)-catechin; Epi, (-)-epicatechin; B2, procyanidin B2; Caf, caffeic acid; Cum, p-coumaric acid; Que, quercitrin; Myr, myricitrin.

flavonols, and hydroxycinnamic acids have been assayed as copigments. However, the pigment and copigment concentrations and ratios generally tested have been much greater than those actually found in red wines. Besides, the effects of copigmentation on color have been generally interpreted only by means of the bathochromic and hyperchromic shifts affecting the visible absorption spectrum in the original pigment solution (3, 13-15). However, the entire visible spectral curve (380$770 \mathrm{~nm}$ ) has to be considered to obtain a complete definition of color. To our knowledge, this consideration was first postulated by Gonnet (16), but nowadays, influence of copigmentation on color continues being investigated mostly by means of the specific changes in the $\lambda_{\max }$ of the visible absorption spectrum.

In an attempt to advance in the knowledge of the contribution of copigmentation to the color of red wines, the purpose of this work was to study the effects of seven phenolic copigments on the color of malvidin 3-glucoside in model wine solutions by using anthocyanin/copigment molar ratios closer to those that may exist in wines. Color changes have been studied by tristimulus colorimetry, which offers an objective measurement of color because it is based on the consideration of the whole visible spectrum, and allows the real assessment of color to be obtained.

\section{MATERIALS AND METHODS}

Standards. The copigments (+)-catechin, (-)-epicatechin, caffeic acid, $p$-coumaric acid, and quercitrin were purchased from Sigma Chemical Co. (St. Louis, MO), and myricitrin was obtained from Extrasynthese (Genay, France). Procyanidin dimer B2 was isolated in the laboratory from seeds of Vitis vinifera grapes, as described by Pascual-Teresa et al. (17).

The pigment malvidin 3-glucoside (Mv3g) was also isolated in the laboratory from skins of $V$. vinifera red grapes of Tempranillo variety, by extraction with acidic methanol and further purification by semipressure liquid chromatography using a reversed-phase column, as described elsewhere (18).

Preparation of Model Solutions. Seven copigmented solutions and a reference solution were prepared in a wine-like medium containing $5 \mathrm{~g} / \mathrm{L}$ tartaric acid in $12 \%$ ethanol and adjusted to $\mathrm{pH} 3.6$ with $\mathrm{NaOH}$. The reference solution contained $200 \mathrm{mg} / \mathrm{L}(0.41 \mathrm{mM})$ of $\mathrm{Mv} 3 \mathrm{~g}$. Copigmented solutions contained the same anthocyanin concentration $(0.41 \mathrm{mM})$ and the corresponding copigment using a copigment/pigment molar ratio of 1:1. All the preparations $(14 \mathrm{~mL})$ were stored closed, in darkness at $25^{\circ} \mathrm{C}$, and samples for the analysis were taken periodically over 60 days.

HPLC-DAD Analysis. A Hewlett-Packard 1100 equipment (Agilent Technologies, Waldbronn, Germany) provided with a quaternary pump, automatic injector, diode array detector, and data treatment station was used. A Spherisorb ODS C18 column $(4.6 \mathrm{~mm} \times 150 \mathrm{~mm}$, $3 \mu \mathrm{m}$ particle size) (Waters, Milford, MA) thermostated at $35^{\circ} \mathrm{C}$ was used. Solvents were (A) $0.1 \%$ trifluoroacetic acid, and (B) $100 \%$ HPLCgrade acetonitrile. The elution profile was as follows: $0 \mathrm{~min}, 90 \% \mathrm{~A}$, $10 \% \mathrm{~B} ; 5-10 \mathrm{~min}, 85 \% \mathrm{~A}, 15 \% \mathrm{~B} ; 15 \mathrm{~min}, 82 \% \mathrm{~A}, 18 \% \mathrm{~B} ; 40 \mathrm{~min}$, $65 \%$ A, $35 \% \mathrm{~B} ; 45-50 \mathrm{~min}, 50 \% \mathrm{~A}, 50 \% \mathrm{~B}$; 55-60 min, $90 \% \mathrm{~A}, 10 \%$ $\mathrm{B}$. The flow-rate was $0.5 \mathrm{~mL} / \mathrm{min}$ and the injection volume $100 \mu \mathrm{L}$. UV-vis spectra were recorded from 250 to $770 \mathrm{~nm}$ with a bandwith of $2 \mathrm{~nm}$. The preferred detection wavelengths were 280, 330, 370,430, and $520 \mathrm{~nm}$.

LC-MS Analysis. LC-MS analyses were performed using a Finnigan LCQ MS detector (Thermoquest, San Jose, CA) equipped with an API source, using an electrospray ionization (ESI) interface. The HPLC system was connected to the probe of the mass spectrometer via the UV cell outlet. Both the sheath and the auxiliary gas were a mixture of nitrogen and helium. The sheath gas flow was $80 \mathrm{~L} / \mathrm{min}$ and the auxiliary gas flow $20 \mathrm{~L} / \mathrm{min}$. The capillary voltage was $26 \mathrm{~V}$ and the capillary temperature $195{ }^{\circ} \mathrm{C}$. Spectra were recorded in positive ion mode between $\mathrm{m} / \mathrm{z}, 150$ and 2000. The mass spectrometer was programmed to do a series of three consecutive scans: a full mass, an $\mathrm{MS}^{2}$ scan of the most abundant ion in the full mass, and an $\mathrm{MS}^{3}$ of the most abundant ion in the $\mathrm{MS}^{2}$. The normalized energy of collision was $45 \%$.

Colorimetric Measurements. The absoption spectra were recorded using a Hewlett-Packard UV-visible HP 8452 spectrophotometer (Palo Alto, CA), using $5 \mathrm{~mm}$ path length glass cells. The whole visible spectrum $(380-770 \mathrm{~nm})$ was recorded $(\Delta \lambda=2 \mathrm{~nm})$, and Illuminant $\mathrm{D}_{65}$ and $10^{\circ}$ Observer were used in the calculus. The CIELAB parameters $\left(L^{*}, a^{*}, b^{*}, C^{*}{ }_{\mathrm{ab}}, h_{\mathrm{ab}}\right)$ and a CIELUV parameter $\left(s^{*}{ }_{\mathrm{uv}}\right)$ were determined by using the original software CromaLab (19), following the recommendations of the Commission International de L'Éclairage (20). Saturation, $s^{*}$ uv, was included in the colorimetric analysis because it is considered the best correlation for the visually perceived saturation, and CIELAB space cannot define a similar correlation (21).

Copigmentation Measurement. The magnitude of the copigmentation was estimated by comparison of the absorbance at $520 \mathrm{~nm}$ from the Mv3g solution $\left(A_{0}\right)$ and the absorbance at $520 \mathrm{~nm}$ from the Mv3g solution copigmented with the different phenolic compounds $(A)$ at each time point and expressed as the percentage $\left[\left(A-A_{0}\right) / A_{0}\right] \times 100(22)$.

\section{RESULTS AND DISCUSSION}

Copigmentation Effect. The magnitude of the copigmentation effect has been found to be dependent on the nature of the copigment, and can be measured by the ratio $\left(A-A_{0}\right) / A_{0}(22)$. As illustrated in Figure 1, an increase in this ratio (i.e., existence of a copigmentation effect) was found to occur in all the solutions despite the low copigment/pigment molar ratio used, although its magnitude was different depending on the com- 
pound used as copigment. Thus, until the day 20 of storage, flavan-3-ols appeared as the less effective copigments, procyanidin B2 being even worse than monomeric flavanols, as previously observed by Berke and Freitas (23). In contrast, flavonols behaved as the best ones, increasing absorptivity between 45 and $55 \%$. They also induced the most statistically significant bathochromic shift in $\lambda_{\max }(9 \mathrm{~nm}$ with myricitrin, and $5 \mathrm{~nm}$ with quercitrin). Flavonols have been considered to be the best copigments in red wines due to their planar polyphenolic nucleus, which can tightly stack onto anthocyanins $(9,24-26)$. On the other hand, flavan-3-ols are comparatively poor cofactors $(3,27)$, and $(-)$-epicatechin has been indicated to associate more strongly to the anthocyanin than its stereoisomer catechin, due to the location of the 3-hydroxy group and the B-ring (28). Thus, in catechin, these groups are situated on different sides of the A-ring plane, while in epicatechin, both groups are situated on the same side of the plane, which allows it a closer approach to the anthocyanin. However, in our test conditions, no differences have been found in the effectiveness of these stereoisomers as copigments of $\mathrm{Mv} 3 \mathrm{~g}$, and with the time of storage, $(+)$-catechin has shown to provide greater enhancement on color than (-)-epicatechin. The fact that procyanidin $\mathrm{B} 2$ has been shown to be a worse copigment than its constituent monomers might be explained by its greater size which would make its approach to the flavylium ion more difficult. With respect to hydroxycinnamic acids, Darias-Martin et al. (29) also concluded these compounds to be better copigments than flavan-3-ols, since the addition of caffeic acid to red wine enhanced color more than the addition of catechin.

Along storage, a progressive hyperchromic shift was produced in the solutions containing hydroxycinnamic acids and catechins. This observation suggests that the copigmentation is not necessarily an immediate effect, but apparently it is established more gradually for the less effective copigments. Furthermore, the stability of the copigmentation complexes was also different according to the cofactor. Thus, in the case of flavonols, a fall in the ratio $\left(A-A_{0}\right) / A_{0}$ was produced after 3 weeks of storage, being specially marked for quercitrin. It is interesting to note that, along the storage period, a significant loss of quercitrin was produced (around 34\%), while the contents of myricitrin in the solutions hardly decreased. The different changes in the concentration of both flavonols would influence the extent of the copigmentation process and explain the greater decrease of the ratio $\left(A-A_{0}\right) / A_{0}$ in the solutions containing quercitrin compared to myricitrin.

At the end of the storage period, Mv3g solutions containing hydroxycinnamic acids showed a higher hyperchromic shift than solutions containing flavonols. This shift should not be explained by an effect of copigmentation and its possible influence on the stability of the anthocyanin, but by the formation of new pigments, which would also influence the color of the solutions at this stage.

Effects of Copigmentation on Color. The copigmentation phenomenon is usually assessed by looking at the changes in $\lambda_{\max }$ of the visible absorption spectrum. However, for a real colorimetric characterization, modifications in the whole visible spectrum should be taken into account. In this study, the total color change has been monitored in the CIELUV and CIELAB color space systems. Figure $\mathbf{2}$ shows the color changes produced in all the solutions. It can be observed that the changes were more marked when flavonols were used as copigments. At the beginning of the assay (day 0) and compared with the Mv3g reference solution, the addition of copigments induced a decrease in lightness $L^{*}$ and an increase in saturation $s^{*}{ }_{\mathrm{uv}}$ and chroma
$C^{*}$ ab, with the exception of the solution containing quercitrin, which decreased chroma values despite its increase in visual saturation. This observation suggests that, during the first days of storage, the high fall in lightness produced by the addition of quercitrin influenced saturation $\left(s_{\mathrm{u} v}^{*}=C^{*}{ }_{\mathrm{uv}} / L^{*}\right)$ more than the fall in chroma. However, from the day 12 of storage, chroma values of the anthocyanin solutions copigmented with quercitrin increased and, as the other copigments, they got greater than those of the Mv3g reference solution. Therefore, the hyperchromic effect produced by copigmentation is connected with a darkening of the anthocyanin solution and with a greater quantity of color and visual saturation. Besides, these effects are more marked with the time of storage. These observations indicate that the presence of the copigments helps to maintain the color in anthocyanin solutions during the storage.

With respect to the hue $h_{\mathrm{ab}}$, flavonols shifted the color of the anthocyanin solutions toward red-purple, whereas the other copigments shifted it toward a more red-orange color. Along the storage period, flavan-3-ols have shown to be the least protective cofactors against browning of the anthocyanin solution, since the hue of the preparations containing these compounds suffered a sharp increase to more red-orange zones in the second month of storage. This greater browning could be related to the formation of yellow pigments, with maximum absorption in the visible region between 430 and $470 \mathrm{~nm}$, as further discussed.

With respect to the solutions containing hydroxycinnamic acids, a slight decrease in the hue values was first produced (i.e., a shift toward a more bluish color), which indicates that some copigmentation occurs. However, from day 30 of storage, hue angles exceeded those of the Mv3g solutions. This fact may be due to the formation of new pigments with pyranoanthocyanin structure, as it will be discussed later.

Color differences, $\Delta E^{*}{ }_{\mathrm{ab}}=\left[\left(\Delta L^{*}\right)^{2}+\left(\Delta a^{*}\right)^{2}+\left(\Delta b^{*}\right)^{2}\right]^{1 / 2}$, between Mv3g solutions with and without copigments, were also calculated (Table 1). In general, the eye is able to discriminate two colors when $\Delta E^{*}$ ab $\geq 1(30,31)$, but when the wine is observed through standardized winetasting glasses, the color discrimination is worse and the acceptable tolerance for the human eye in distinguishing the color of wines is 3.0 CIELAB units (32). Color differences between anthocyanin solutions copigmented with flavonols and Mv3g control solutions were always easily detectable by the human eye, since $\Delta E^{*}$ ab values were always much higher than 3 units; specifically, they were between 7.67 and 23.30 for quercitrin and between 6.90 and 21.77 for myricitrin. Therefore, copigmentation between flavonols and Mv3g led to very significant color changes in the anthocyanin solution from the earliest moment of contact copigment-pigment. However, these effects were not so noticeable for the other copigments. Thus, color differences between Mv3g solutions copigmented with and without hydroxycinnamic acids would not be detectable $\left(\Delta E^{*}{ }_{\mathrm{ab}} \geq 3\right)$ until the third day of storage, and until the day 13 for the solutions copigmented with catechins. Procyanidin B2 was the copigment leading to less color changes in the Mv3g solution, which would not be appreciated until the day 23 of storage.

Chemical Changes in the Solutions during Storage. Figure 3 shows the changes in the concentration of Mv3g in all the solutions assayed along the 2 months of storage. It can be observed that the presence of the copigments at the copigment/ pigment molar ratio used (1:1) does not impede the loss of the anthocyanin, whose decrease is roughly similar in all the solutions, but the one containing $p$-coumaric acid. This latter compound has shown to behave as a protective cofactor against 

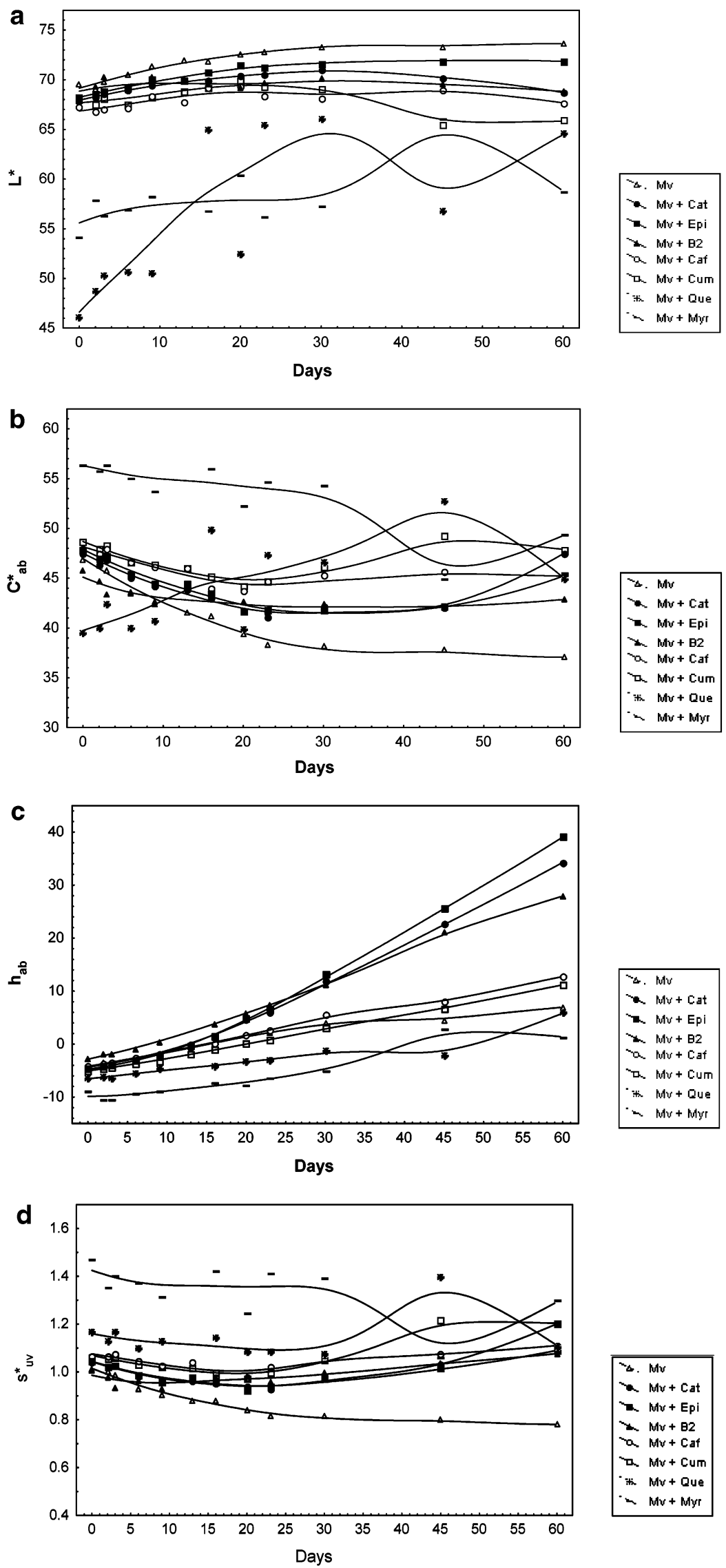

Figure 2. (a) Changes in the lightness, $L^{*}$, of the model solutions containing malvidin 3 -glucoside and different copigments during storage at $25^{\circ} \mathrm{C}$. (b) Changes in the chroma, $\mathrm{C}_{\mathrm{ab}}^{*}$, of the model solutions containing malvidin 3-glucoside and different copigments during storage at $25^{\circ} \mathrm{C}$. (c) $\mathrm{Changes}$ in the hue angle, $h_{\mathrm{ab}}$, of the model solutions containing malvidin 3-glucoside and different copigments during storage at $25^{\circ} \mathrm{C}$. (d) $\mathrm{Changes}$ in the saturation, $s^{*}{ }_{u v}$, of the model solutions containing malvidin 3-glucoside and different copigments during storage at $25^{\circ} \mathrm{C}$. Abbreviations: Cat, (+)-catechin; Epi, (-)-epicatechin; B2, procyanidin B2; Caf, caffeic acid; Cum, p-coumaric acid; Que, quercitrin; Myr, myricitrin. 
Table 1. Color Differences $\left(\Delta E^{*}\right.$ ab $)$ between the Reference Solution of Malvidin 3-Glucoside and the Solutions Added with Different Copigments during Storage at $25^{\circ} \mathrm{C}^{a}$

\begin{tabular}{rrrrrrrr} 
& \multicolumn{7}{c}{ copigment } \\
\cline { 2 - 7 } day & CAT & EPI & \multicolumn{1}{c}{ B2 } & CAF & CUM & QUE & MYR \\
\hline 0 & 1.69 & 1.66 & 1.02 & 2.47 & 2.39 & 23.30 & 18.28 \\
2 & 0.97 & 1.57 & 1.27 & 2.66 & 2.51 & 22.08 & 17.60 \\
3 & 1.63 & 1.72 & 1.58 & 3.56 & 3.03 & 20.23 & 19.44 \\
6 & 2.11 & 2.31 & 1.95 & 4.52 & 4.29 & 20.46 & 18.94 \\
9 & 2.42 & 2.21 & 1.40 & 4.62 & 4.86 & 20.58 & 17.69 \\
13 & 3.09 & 3.53 & 2.80 & 6.10 & 5.14 & 22.38 & 18.29 \\
16 & 2.86 & 2.78 & 1.92 & 3.86 & 4.84 & 9.82 & 20.32 \\
20 & 3.72 & 3.33 & 2.72 & 5.32 & 5.61 & 19.06 & 17.28 \\
23 & 4.53 & 4.95 & 5.92 & 7.78 & 7.41 & 9.82 & 21.77 \\
30 & 7.15 & 7.61 & 4.08 & 8.59 & 9.04 & 8.47 & 20.26 \\
45 & 13.54 & 15.21 & 9.18 & 9.60 & 14.36 & 19.49 & 6.90 \\
60 & 21.77 & 22.88 & 13.03 & 10.53 & 13.44 & 7.67 & 16.05
\end{tabular}

${ }^{a}$ CAT, (+)-catechin; EPI, (-)-epicatechin; B2, procyanidin B2; CAF, caffeic acid; CUM, p-coumaric acid; QUE, quercitrin; MYR, myricitrin.

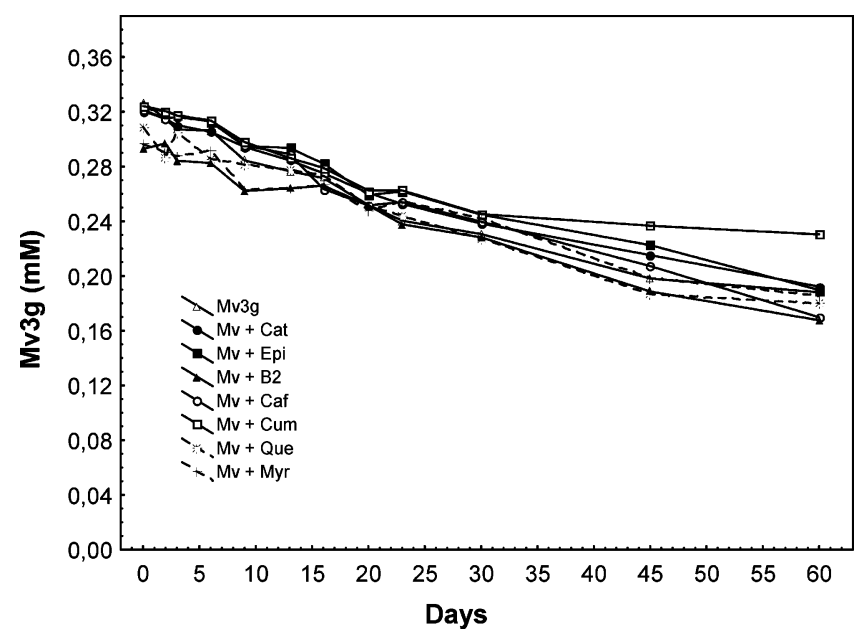

Figure 3. Changes in the concentration (mM) of malvidin 3-glucoside (Mv3g) in solutions containing different copigments. Abbreviations: Cat, (+)-catechin; Epi, (-)-epicatechin; B2, procyanidin B2; Caf, caffeic acid; Cum, p-coumaric acid; Que, quercitrin; Myr, myricitrin.

Mv3g degradation. Obviously, other protective factors also exist in red wines that limit the rate of degradation of anthocyanins, whose decline takes place much slower than it does in model solutions.

Despite the anthocyanin decrease, the color is better maintained in the solutions that contain copigments, as commented above. This improvement in color stability may be initially explained by the existence of a copigmentation process and the subsequent protection of the anthocyanin against hydration. The existence of copigmentation is clear in the solutions containing flavonols, where the characteristic hyperchromic and bathochromic shifts are rapidly observed. However, for the remaining copigments, the color maintenance and further changes in the hue of the solutions during storage could also be explained by the formation of new pigments. Thus, in the solutions containing hydroxycinnamic acids, the appearance of a new pigment was observed from 2 weeks of storage in each solution. In the solution containing caffeic acid, that pigment (peak a in the chromatogram of Figure 4) showed $\lambda_{\max }$ in the visible region at $508 \mathrm{~nm}$, whereas in that containing $p$-coumaric acid, $\lambda_{\max }$ of the new pigment was $502 \mathrm{~nm}$. The mass spectra of those peaks showed positive molecular ions at $\mathrm{m} / \mathrm{z} 625$ and $\mathrm{m} / \mathrm{z} 609$, respectively. The ion at $m / z 625$ released an $\mathrm{MS}^{2}$ fragment at $m / z 463$ ( $-162 \mathrm{amu}$, loss of a glucose moiety), which led to

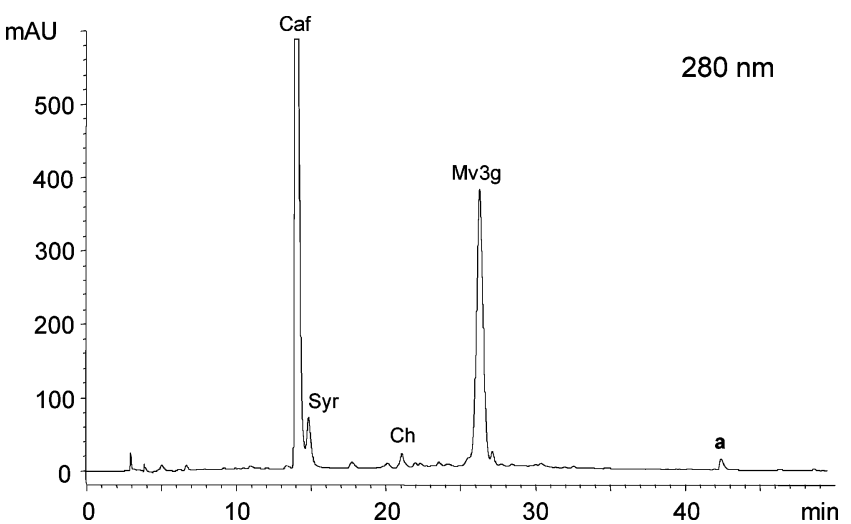

Figure 4. HPLC chromatogram recorded at $280 \mathrm{~nm}$ of Mv3g solutions containing caffeic acid at the end of the storage period. Abbreviations: Caf, caffeic acid; Syr, syringic acid; Mv3g, malvidin 3-glucoside; Ch, $Z$-chalcone of Mv3g. Peak a is a newly formed pigment.

major $\mathrm{MS}^{3}$ fragment ions at $\mathrm{m} / \mathrm{z} 447$ and 431 (successive loss of two hydroxyl residues). The ion at $\mathrm{m} / \mathrm{z} 609$ observed in the solution containing $p$-coumaric acid gave a major $\mathrm{MS}^{2}$ fragment at $m / z, 447(-162 \mathrm{amu})$ and a further $\mathrm{MS}^{3}$ fragment at $\mathrm{m} / \mathrm{z}, 431$ $(-16 \mathrm{amu})$. In accordance with their UV and mass spectra, these pigments were identified as the 4-vinylcatechol and 4-vinylphenol pyranoanthocyanin adducts of $\mathrm{Mv} 3 \mathrm{~g}$, respectively (structure 6 in Figure 8). The formation of such kind of pigments in red wines and model wine systems has been previously described $(33-35)$. Their formation in the solutions studied here would take place according to the mechanism described by Schwarz et al. (36): a bond would be established between the C-4 position of Mv3g and the C-2 position of the hydroxycinnamic acid leading to an intermediate carbenium compound, which can be trapped intramolecularly by the phenolic hydroxyl group of $\mathrm{Mv} 3 \mathrm{~g}$ to form the new pyran ring. Further decarboxylation and oxidation of these pyran moieties would be the final steps. Although the content of these new pigments in the solutions is not very high in comparison with the anthocyanin, and the molar extinction coefficients $(\epsilon)$ of their flavylium forms and Mv3g are very similar (37), they are expected to contribute to the color of the solutions (38). This is because they have a hydration constant $\left(K_{\mathrm{h}}\right)$ smaller than that of the anthocyanin (39), and therefore, they occur in much greater proportion in colored forms than the anthocyanin at weak acidic conditions, as those existing in the model solutions and red wines.

In addition to these new pigments, two more new products appeared in the solutions containing hydroxycinnamic acids (Figure 4). Peak "Syr" was identified as syringic acid, on the basis of its UV spectra $\left(\lambda_{\max }=276 \mathrm{~nm}\right)$, retention time, and mass spectrum (molecular ion at $m / z$ 199). It would result from the moiety corresponding to the B-ring released after degradation of Mv3g (40-42). Peak "Ch" was detected from the first day of assay, and it was assigned to the chalcone form of Mv3g, according to its UV ( $\lambda_{\max }$ at $350 \mathrm{~nm}$ ) and MS spectra (positive ion signals at $m / z 511$ and 349 comparable to the Mv3g chalcone form and its aglycon, respectively). It can be assumed to be the $Z$-chalcone form that, due to its slow reversion, would not be shifted toward the flavylium form during the chromatographic elution, as happens with other forms of the anthocyanin. These two products would not contribute to the color of the solutions, since they showed hardly or no absorption in the visible region of the spectrum.

The formation of new compounds was also observed in the solutions containing flavan-3-ols (Figure 5). Some of them had $\lambda_{\max }$ in the visible region between 430 and $470 \mathrm{~nm}$ and, 

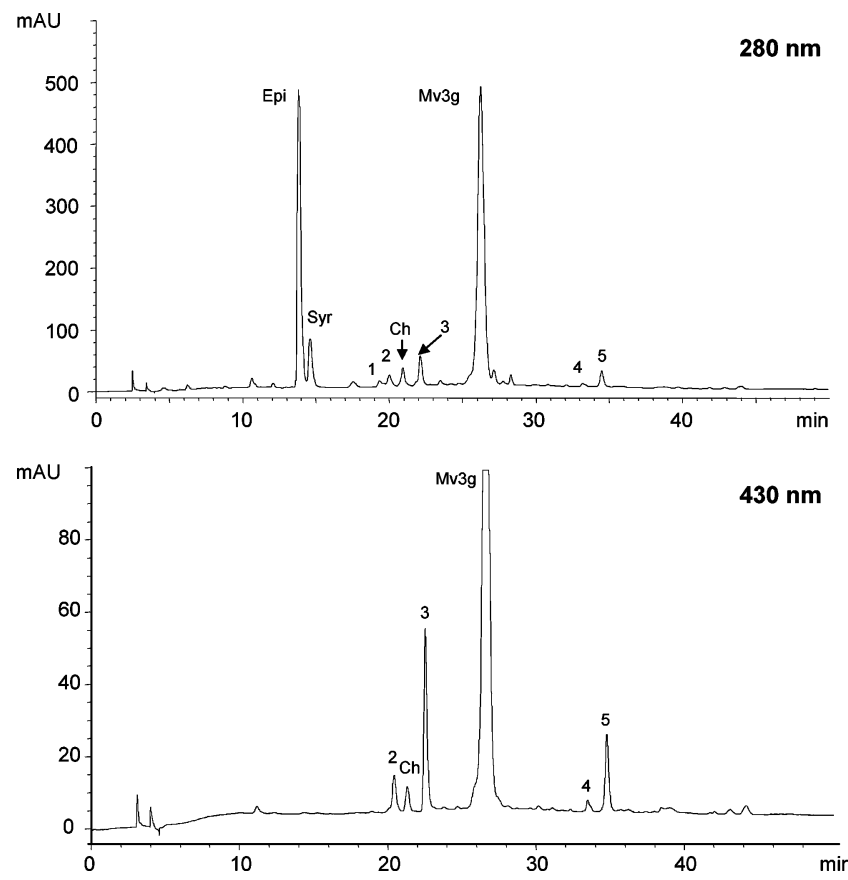

Figure 5. HPLC chromatograms of Mv3g solutions containing (-)epicatechin recorded at 280 and $430 \mathrm{~nm}$ at the end of the storage period. Abbreviations: Epi, epicatechin; Syr, syringic acid; $M v 3 g$, malvidin 3-glucoside; Ch, Z-chalcone of Mv3g.

therefore, contributed to yellowish hues. Peak 2 had $\lambda_{\max }$ at 438 $\mathrm{nm}$ and a molecular ion at $\mathrm{m} / \mathrm{z}, 617$, with major $\mathrm{MS}^{2}$ fragment ions at $m / z, 465(-152 \mathrm{amu}$, retro Diels-Alder fission of a catechin moiety) and 447 ( $-170 \mathrm{amu}$, further loss of water). The formation of this pigment was already found in previous works of our group (42). Specifically, it would correspond to the xanthylium pigment called NJ2 (structure 1 in Figure 8) and identified by Es-Safi et al. $(43,44)$. This pigment is derived from the condensation between two catechin units through glyoxylic acid followed by an oxidation step. The glyoxylic acid would be formed in the solutions from the oxidation of tartaric acid (45). Peak 3 showed $\lambda_{\max }$ at $438 \mathrm{~nm}$ and a molecular ion at $\mathrm{m} / \mathrm{z}$ 453, with major $\mathrm{MS}^{2}$ fragment ions at $\mathrm{m} / \mathrm{z} 301(-152$ amu), 283 ( $-170 \mathrm{amu})$, and 257 ( $-196 \mathrm{amu})$. This compound was also previously found by our group in model solutions (42) and attributed to a xanthylium derivative (structure 2 in Figure 8) similar to peak 2, where one of the catechin units was substituted by phloroglucinol. The phloroglucinol residue might derive from the A-ring of Mv3g following anthocyanin breakdown (42). Peaks 4 and 5 had $\lambda_{\max }$ in the visible region at 460 and $430 \mathrm{~nm}$, respectively, and their mass spectra showed molecular ions at $\mathrm{m} / \mathrm{z} 645$ (with a major $\mathrm{MS}^{2}$ fragment ion at $m / z$ 493) and $m / z 633$ (major $\mathrm{MS}^{2}$ fragment ions at $\mathrm{m} / \mathrm{z} 435$, 453), respectively. These data suggest that they would correspond to xanthylium pigments $\mathrm{NJ} 3$ and NJ3' (structure 1 in Figure 8) also described by Es-Safi et al. (44).

In addition to the yellow pigments, the formation of other products was also found in the solutions copigmented with flavan-3-ols. Besides syringic acid and the chalcone form of the anthocyanin, another colorless compound (peak 1 in Figure 5) was observed showing $\lambda_{\max }$ at $274 \mathrm{~nm}$, and a molecular ion at $m / z$ 637, with major $\mathrm{MS}^{2}$ fragment ions at $\mathrm{m} / \mathrm{z} 467$ and 485 . These data suggest that it could be a colorless carboxymethinelinked dimer (structure 3 in Figure 8), resulting from the condensation of two catechin units mediated by glyoxylic acid. This product would be the precursor of the xanthylium pigments (45).

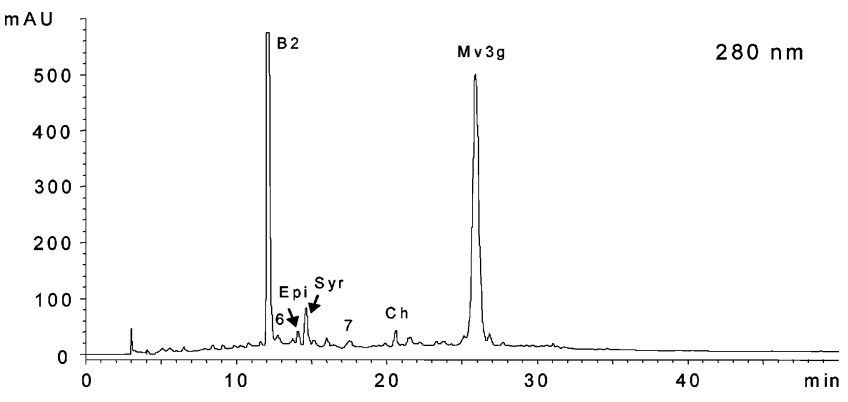

Figure 6. HPLC chromatogram recorded at $280 \mathrm{~nm}$ of the Mv3g solution containing procyanidin $\mathrm{B} 2$ at the end of the storage period. Abbreviations: Epi, epicatechin; B2, procyanidin B2; Syr, syringic acid; Mv3g, malvidin 3-glucoside; Ch, Z-chalcone of Mv3g.

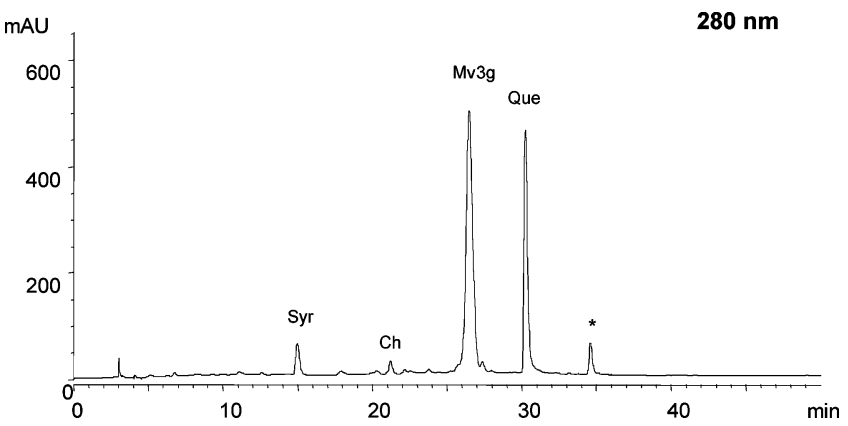

Figure 7. HPLC chromatogram recorded at $280 \mathrm{~nm}$ of the Mv3g solution containing quercitrin at the end of the storage period. Abbreviations: Que, quercitrin; Mv3g, malvidin 3-glucoside; Syr, syringic acid; Ch, Z-chalcone of Mv3g. The peak marked with an asterisk corresponds to an unidentified product derived from quecitrin.

Compared to the solutions containing monomeric flavan-3ols, hardly any formation of new pigments was observed in the solutions containing procyanidin B2 (Figure 6). Thus, peak 6 showed $\lambda_{\max }$ at $440 \mathrm{~nm}$ and a molecular ion at $\mathrm{m} / \mathrm{z}$ 741, with released $\mathrm{MS}^{2}$ fragment ions at $m / z$ 527, 451, 289, and 375. These data suggest that it could be a xanthylium pigment similar to peak 3 but showing phloroglucinol and procyanidin B2 residues (structure 5 in Figure 8). Peak 7 showed visible $\lambda_{\max }$ at 460 $\mathrm{nm}$ and a molecular ion at $m / z, 1193$, with a major $\mathrm{MS}^{2}$ fragment ion at $m / z, 903$, which released $\mathrm{MS}^{3}$ fragment ions at $\mathrm{m} / \mathrm{z}$ 451, 595 , and 585. On the basis of these mass spectral characteristics and the previous findings in catechin solutions (42-44), this compound can be assigned to a xanthylium pigment similar to peak 2, but containing two procyanidin B2 moieties (structure 4 in Figure 8). Among the colorless compounds, the presence of small amounts of (-)-epicatechin was also detected in the solutions, indicating that a breakdown of the interflavanic linkage of the dimer occurs.

In the solutions added with flavonols, the formation of colorless compounds was detected, specifically, the chalcone form of $\mathrm{Mv} 3 \mathrm{~g}$ and syringic acid derived from the degradation of the anthocyanin. In the last month of the assay, another product showing $\lambda_{\max }$ at $340 \mathrm{~nm}$ was also detected in the solution added with quercitrin (Figure 7). Taking into account its UV spectrum similar to flavonols and the important decrease produced in quercitrin levels at this stage of storage, it is supposed to derive from the degradation of this latter. However, no further discussion can be made about its structure, since no good mass spectrum could be obtained in the conditions of ionization used.

In conclusion, the effectiveness of seven phenolic compounds (catechin, epicatechin, procyanidin $\mathrm{B} 2$, caffeic acid, $p$-coumaric acid, myricitrin, and quercitrin) to act as copigments of $\mathrm{Mv} 3 \mathrm{~g}$ 
1

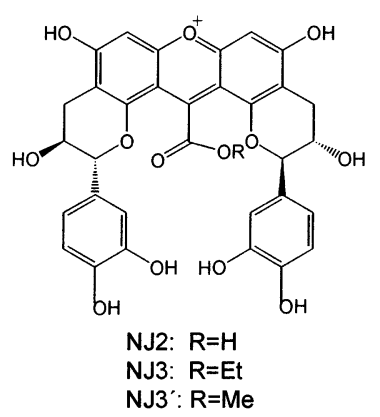

NJ3: $R=E t$

NJ3': R=Me

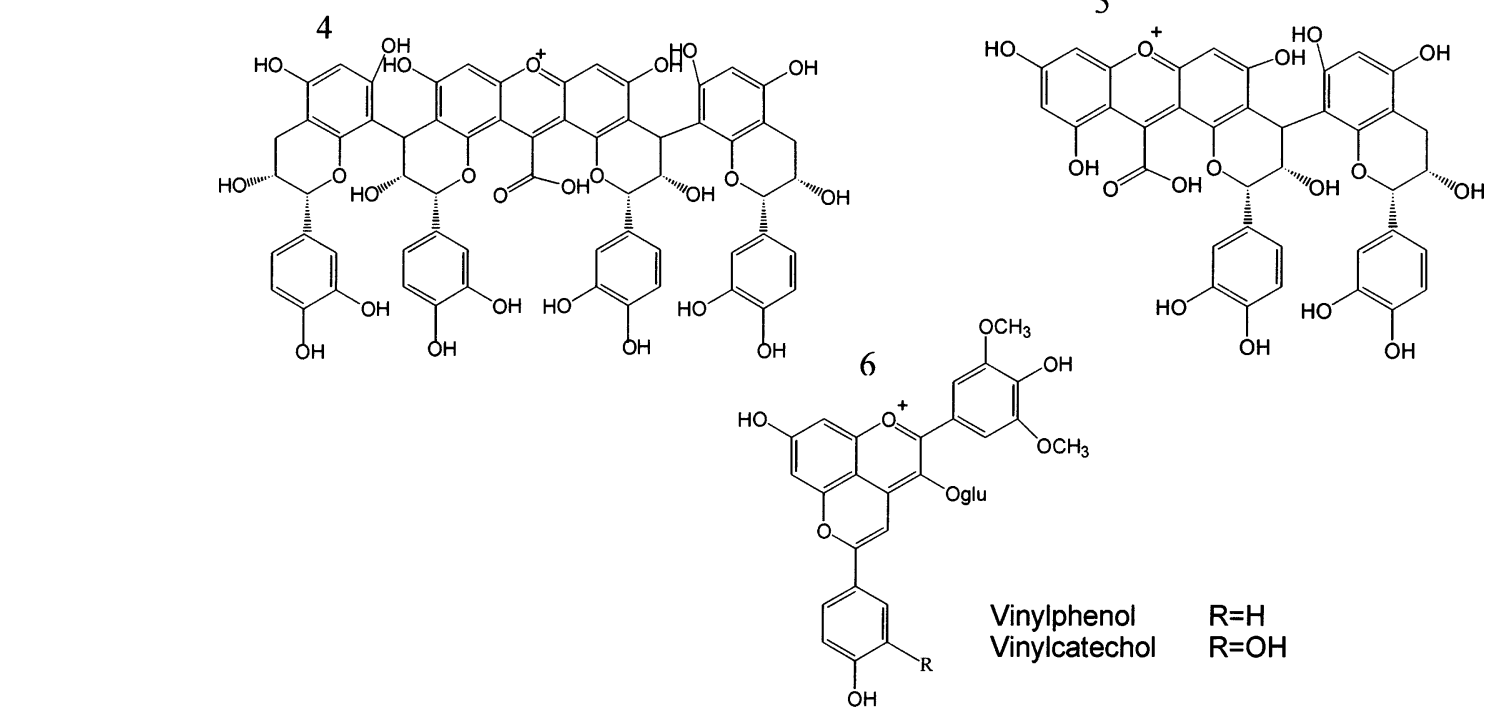

2<smiles>[Y]c1cc2[o+]c3cc(O)cc(O)c3c(C(=O)O)c2c2c1C[C@H](O)[C@H](c1ccc(O)c(O)c1)O2</smiles>

3

5

Figure 8. Suggested structures of different compounds detected in the model solutions. (1) Xanthylium pigments previously identified by Es-Safi et al. $(43,44)$; (2) proposed structure for a xanthylium pigment containing catechin and phloroglucinol residues; (3) colorless dimer obtained by condensation of flavanols mediated by glyoxylic acid (45); (4) xanthylium pigment containing two procyanidin B2 residues; (5) xanthylium pigment containing procyanidin B2 and phloroglucinol residues; and (6) pyranoanthocyanins derived from malvidin 3-glucoside $(34,36)$.

in model wine solutions using a copigment/pigment molar ratio of 1:1 has been studied. Despite their relatively low concentration, all of them were able to induce an increase in the ratio $(A$ $\left.-A_{0}\right) / A_{0}$, characteristic of a copigmentation process. The use of tristimulus colorimetry constituted a suitable alternative to the measurements classically used to evaluate the copigmentation process based on the shifts at maximum wavelength in the visible spectrum of the anthocyanin. Copigmentation was revealed by a decrease in lightness $L^{*}$ and an increase in saturation $s^{*}{ }_{\mathrm{uv}}$, chroma $C^{*}{ }_{\mathrm{ab}}$, and hue $h_{\mathrm{ab}}$. As expected, relevant differences existed among the different compounds assayed regarding their efficiency as anthocyanin copigments. In the conditions of the assay, color differences $\left(\Delta E^{*}{ }_{\mathrm{ab}}\right)$ between Mv3g control and the solutions copigmented with flavonols were detectable by the human eye $\left(\Delta E^{*}\right.$ ab $\left.\geq 3\right)$ since the moment of their addition. Changes induced by hydroxycinnamic acids were not detectable until the third day of storage, whereas catechins and procyanidin B2 required about 2 and 3 weeks, respectively, suggesting that the interactions between anthocyanin and copigments were apparently established more gradually for the less effective ones. With the time of storage, other kind of reactions leading to the formation of new pigments also occurred, especially in the anthocyanin solutions added with hydroxycinnamic acids and flavan-3-ols, which also contributed to the changes observed in the color of the solutions at the end of the period of storage (60 days).

\section{LITERATURE CITED}

(1) Brouillard, R. Anthocyanins as Food Colors; Academic Press: London, 1982; pp 1-38.

(2) Mazzaracchio, P.; Pifferi, P.; Kindt, M.; Munyaneza, A.; Barbiroli, G. Interactions between anthocyanins and organic food molecules in model systems. Int. J. Food Sci. Technol. 2004, $39,53-59$.

(3) Liao, H.; Cai, Y.; Haslam, E. Polyphenol interactions. Anthocyanins: Co-pigmentation and colour changes in red wines. $J$. Sci. Food Agric. 1992, 59, 299-305.

(4) Dangles, O.; Saito, N.; Brouillard, R. Anthocyanin intramolecular copigment effect. Phytochemistry 1993, 34, 119-124.

(5) Figuereido, P.; George, F.; Tatsuzawa, F.; Toki, K.; Saito, N.; Brouillard, R. New features of intramolecular copigmentation by acylated anthocyanins. Phytochemistry 1999, 51, 125-132.

(6) George, F.; Figuereido, P.; Toki, K.; Tatsuzawa, F.; Saito, N.; Brouillard, R. Influence of trans-cis isomerisation of coumaric acid substituents on colour variance and stabilisation in anthocyanins. Phytochemistry 2001, 57, 791-795.

(7) Mazza, G.; Brouillard, R. The mechanism of co-pigmentation of anthocyanins in aqueous solutions. Phytochemistry 1990, 29, 1097-1102.

(8) Asen, S.; Stewart, R. N.; Norris, K. H. Co-pigmentation of anthocyanins in plant tissues and its effects on color. Phytochemistry 1972, 11, 1139-1144.

(9) Baranac, J. M.; Petranovic, N. A.; Dimitric-Markovic, J. M. Spectrophotometric study of anthocyanin copigmentation reactions. J. Agric. Food Chem. 1996, 44, 1333-1336. 
(10) Santos-Buelga, C.; Bravo-Haro, S.; Rivas-Gonzalo, J. C. Interactions between catechin and malvidin-3-monoglucoside in model solutions. Z. Lebensm.-Unters. Forsch. 1995, 201, 269-274.

(11) Dimitric-Markovic, J. M.; Petranovic, N. A.; Baranac, J. M. The copigmentation effect of sinapic acid on malvin: A spectroscopic investigation on colour enhancement. J. Photochem. Photobiol. B 2005, 78, 223-228.

(12) Boulton, R. The copigmentation of anthocyanins and its role in the color of red wine: A critical review. Am. J. Enol. Vitic. 2001, $52,67-87$.

(13) Mirabel, M.; Saucier, C.; Guerra, C.; Glories, Y. Copigmentation in model wine solutions: Occurrence and relation to wine aging. Am. J. Enol. Vitic. 1999, 50 (2), 211-218.

(14) Dimitric-Markovic, J.; Petranovic, N. A.; Baranac, J. M. A spectrophotometric study of the copigmentation of malvin with caffeic and ferulic acids. J. Agric. Food Chem. 2000, 48, 55305536.

(15) Eiro, M. J.; Heinonen, M. Anthocyanin color behavior and stability during storage: Effect of intermolecular copigmentation. J. Agric. Food Chem. 2002, 50, 7461-7466.

(16) Gonnet, J.-F. Colour effects of copigmentation of anthocyanins revisited-1. A colorimetric definition using CIELAB scale. Food Chem. 1998, 63(3), 409-415.

(17) Pascual-Teresa, S.; Santos-Buelga, C.; Rivas-Gonzalo, J. C. Quantitative analysis of flavan-3-ols in Spanish foodstuffs and beverages. J. Agric. Food Chem. 2000, 48 (11), 5331-5337.

(18) Heredia, F. J.; Francia-Aricha, E. M.; Rivas-Gonzalo, J. C.; Vicario, I. M.; Santos-Buelga, C. Chromatic characterization of anthocyanins from red grapes-I. pH effect. Food Chem. 1998, 63 (4), 491-498.

(19) Heredia, F. J.; Álvarez, C.; González-Miret, M. L.; Ramírez, A. CromaLab, análisis de color. Registro General de la Propiedad Intelectual SE-1052-04: Sevilla, Spain, 2004.

(20) CIE. Colorimetry, 2nd ed.; Publication 15.2; CIE: Vienna, Austria, 1986

(21) Wyszecki, G.; Stiles, W. S. Color Science. Concepts and Methods. Quantitative Data and Formulae, 2nd ed.; John Wiley \& Sons: New York, 1982.

(22) Boulton, R. B. A method for the assessment of copigmentation in red wines. Presented at the 47th Annual Meeting of the American Society for Enology and Viticulture. Reno, NV, June 1996.

(23) Berke, B.; De Freitas, V. A. P. Influence of procyanidin structures on their ability to complex with oenin. Food Chem. 2005, 90, 453-460.

(24) Baranac, J. M.; Petranovic, N. A.; Dimitric-Markovic, J. M. Spectrophotometric study of anthocyan copigmentation reactions. J. Agric. Food Chem. 1996, 44, 1333-1336.

(25) Baranac, J. M.; Petranovic, N. A.; Dimitric-Markovic, J. M. Spectrophotometric study of anthocyan copigmentation reactions. 2. Malvin and the nonglycosidized flavone quercetin. J. Agric. Food Chem. 1997, 45, 1694-1697.

(26) Baranac, J. M.; Petranovic, N. A.; Dimitric-Markovic, J. M. Spectrophotometric study of anthocyan copigmentation reactions. 3. Malvin and the nonglycosidized flavone morin. J. Agric. Food Chem. 1997, 45, 1698-1700.

(27) Escribano-Bailón, M. T.; Dangles, O.; Brouillard, R. Coupling reactions between flavylium ions and catechin. Phytochemistry 1996, 41, 1583-1592.

(28) Brouillard, R.; Wigand, M. C.; Dangles, O., Cheminat, A. pH and solvent effects on the copigmentation reaction of malvin with polyphenols, purine and pyrimidine derivatives. J. Chem. Soc., Perkin Trans. 1991, 2, 1235-1241.
(29) Darias-Martín, J.; Carrillo, M.; Díaz, E., Boulton R. B. Enhancement of red wine colour by pre-fermentation addition of copigments. Food Chem. 2001, 73, 217-220.

(30) Gonnet, J. F. Colour effects of copigmentation of anthocyanins revisited-1. A colorimetric definition using CIELAB scale. Food Chem. 1998, 63, 409-415.

(31) Negueruela, A. I.; Echávarri, J. F.; Ayala, F.; Lomas, A. M. Colorimetría en vinos. Zubía monográfico 1995, 7, 151-166.

(32) Martínez, J. A.; Melgosa, M.; Pérez, M. M.; Hita, E.; Negueruela, A. I. Note: Visual and instrumental color evaluation in red wines. Food Sci. Technol. Int. 2001, 7(5), 439-444.

(33) Cameira-dos-Santos, P. J.; Brillouet, J. M.; Cheynier, V.; Moutounet, M. Detection and partial characterization of new anthocyanin-derived pigments in wine. J. Sci. Food Agric. 1996, 70, 204-208.

(34) Fulcrand, H.; Cameira-dos-Santos, P. J.; Sarni-Manchado, P.; Cheynier, V.; Favre-Bonvin, J. Structure of new anthocyaninderived wine pigments. J. Chem. Soc., Perkin Trans. 1996, 1, 735-739.

(35) Monagas, M.; Gomez-Cordoves C.; Bartolome, B. Evolution of polyphenols in red wines from Vitis vinifera $\mathrm{L}$. during aging in the bottle-I. Anthocyanins and pyranoanthocyanins. Eur. Food Res. Technol. 2005, 220, 607-614.

(36) Schwarz, M.; Wabnitz, T.; Winterhalter, P. Pathway leading to the formation of anthocyanin-vinylphenol adducts and related pigments in red wines. J. Agric. Food Chem. 2003, 51, 36823687.

(37) Hakansson, E.; Pardon, K.; Hayasaka, Y.; de Sa, M.; Herderich, $\mathrm{M}$. Structures and colour properties of new red wine pigments. Tetrahedron Lett. 2003, 44, 4887-4891.

(38) Vivar-Quintana, A. M.; Santos-Buelga, C.; Rivas-Gonzalo, J. C. Anthocyanin-derived pigments and colour of red wines. Anal. Chim. Acta 2002, 458, 147-155.

(39) Sarni-Manchado, P.; Fulcrand, H.; Souquet, J. M.; Cheynier, V.; Moutounet, M. Stability and color of unreported wine anthocyanin-derived pigments. J. Food Sci. 1996, 5, 938-941.

(40) Furtado, P.; Figueiredo, P.; Chaves Das Neves, H.; Pina, F. Photochemical and thermal degradation of anthocyanidins. $J$. Photochem. Photobiol., A 1993, 75 (2), 113-118.

(41) Piffaut, B.; Kader, F.; Girardin, M. Comparative degradation pathways of malvidin 3,5 diglucoside after enzimatic and thermal treatments. Food Chem. 1998, 50, 115-120.

(42) Santos-Buelga, C.; Francia-Aricha, E. M.; De Pascual-Teresa, S.; Rivas-Gonzalo, J. C. Contribution to the identification of the pigments responsible for the browning of anthocyanin-flavanol solutions. Eur. Food Res. Technol. 1999, 209, 411-415.

(43) Es-Safi, N.; Le Guernevé, C.; Fulcrand, H.; Cheynier, V.; Moutounet, M. Xanthylium salts formation involved in wine colour changes. Int. J. Food Sci. Technol. 2000, 35, 63-74.

(44) Es-Safi, N.; Le Guernevé, C.; Fulcrand, H.; Cheynier, V.; Moutounet, M. New polyphenolic compounds with xanthylium skeletons formed through reaction between $(+)$-catechin and glyoxylic acid. J. Agric. Food Chem. 1999, 47, 5211-5217.

(45) Fulcrand, H.; Cheynier, V.; Ozsmianski, J.; Moutounet, M. An oxidized tartaric acid residue as a new bridge potentially competing with acetaldehyde in flavan-3-ol condensation. Phytochemistry 1997, 46, 223-227.

Received for review February 15, 2006. Revised manuscript received May 12, 2006. Accepted May 17, 2006.

JF0604586 